Military Technical College Kobry El-Kobbah, Cairo, Egypt

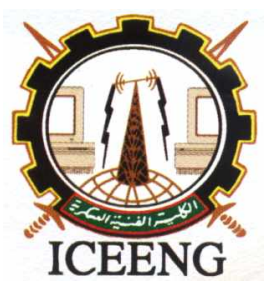

\author{
$6^{\text {th }}$ International Conference \\ on Electrical Engineering \\ ICEENG 2008
}

\title{
Overcoming the CT saturation effect on unit protection schemes using fundamental phase comparison technique
}

By
M. H. Abdel-Aall*
M. A. El-Hadidy*
S. F. Mekhamer**
M. M. Mansour**
M.S.El Samahy $* * *$

\section{$\underline{\text { Abstract: }}$}

Current transformer saturation can cause differential protective relay mal-operation. This paper presents a technique for overcoming current tramsformer saturation problem based on the measurements of the fundamentals' phase-angles of the secondary currents on both sides of the protected object. Mal-operation could be avoided by introducing these measurements to the phase comparison technique which has the ability to distinguish between internal and external faults..

\section{Keywords:}

Current transformer saturation, fundamentals' phase-angles, phase comparison.

* Egyptian Electricity Transmission Company

** Electrical Power and Machines Dept., Ain Shams University

*** Egyptian Armed Forces 


\section{Introduction:}

The main problem that faces the differential protective relay is the saturation of the Current transformer (CT). This situation may result in a false operation of the relay during an external fault; great efforts have been done to avoid the mal-operation of the relay due to this reason. Low Impedance Biased Differential Busbar Protection [1] produces inhibit pulses by a circuit that detects the CT saturation, operate the semiconductor switches. The resultant differential current in the measuring circuit will be a series of narrow spikes, the area under the curve being greatly reduced, so aiding stability.Modal saturation detector was introduced in [2]. The technique transforms the secondary currents of the three phases into space phasors with a blocked backward rotating component. In the unsaturated state their trajectories are circles. The deviation from this characteristic form and hence the inception of CT saturation can be detected in less than 3 milliseconds. Another algorithm [3] is based on the measurement of the power system source impedance seen at the relay location. A technique for discriminating faults in a bus protection zone from those outside the zone has been presented in [4]. This technique uses the sign of the sequence impedances seen by the relays connected to the busbar. The impedances seen by all the relays lie in the third quadrant of the impedance plane when a fault is in the protection zone of the busbar.

The use of artificial neural networks (ANN) to correct CT secondary waveform distortions is explained in [5]. The ANN is trained to achieve the inverse transfer function of iron-core toroidal CT's which are widely used in protective systems. An efficient compensation algorithm capable of converting from a sampled current waveform that is distorted by CT saturation to a compensated current waveform is shown in [6]. A method of analyzing the voltage of bus bar by Wavelet Transform is presented in [7] to detect accurately fault point. It can be distinguished, whether CT is saturated based on the time difference between the time of the fault and the time of differential current. Another compensation algorithm has been introduced in [8]. The saturated current of the CT is analyzed to find out the characterizing features of its harmonics. This harmonic analysis shows that there is a reverse proportionality between the fundamental component of the saturated current and its odd harmonics (for AC saturation). As the CT be more saturated, the fundamental component of its secondary current decreases, while the odd harmonics increase. Depending on these characteristics of the saturated current, this technique suggests an empirical formula for compensating the reduction appears in the CT current due to saturation. The empirical formula adds the increasing harmonic, weighted by a compensating factor, to the decreasing fundamental to reach the actual value of the current as if saturation has not occurred.

This paper introduces the use of the phase comparison (PC) technique to detect the CT saturation, based on the fundamental's phase-angle analysis of the CT's secondary currents, this method has the advantage of overcoming the saturation problem totally by 
monitoring the secondary currents' phase-angles always on-line and introducing this decision to the next step in the relay algorithm enabling the relay to make the right decision.

\section{Phase Comparison Principal}

PC relaying is a kind of differential relaying that compares the phase angles of currents at all terminals of the protected object. For the conditions of a fault within the protected zone (internal fault), the currents entering all the terminals will be in phase. For conditions of a fault outside the zone of protection (external or through fault), or for load flow, the currents entering any one terminal will be $180^{\circ}$ out of phase with the currents entering at least one of the other terminals. The PC relay scheme makes this phase angle comparison and trips the associated breakers for internal faults. Since the terminals of a Transmission Line (TL) are normally many miles apart, some sort of communication channel between the terminals is required to make this comparison possible [9].

The conditions mentioned above are ideal. They seldom, if ever, occur in a real power system. Actually, this is true for a variety of reasons [9] including the following:

1. Current transformer saturation.

2. Phase angle differences between the currents entering both ends of the line as a result of phase angle differences in the driving system voltages.

3. Load and charging currents of the line.

4. Transit time of the communication signal.

5. Unsymmetrical build-up and tail-off times of the receiver.

Figure 1a shows different fault locations on a TL, while figure $1 \mathrm{~b}$ shows the operating and restraint areas for the PC protection scheme

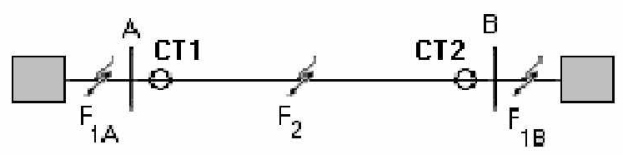

Figure(1a) : Different fault locations on a $T L$ 


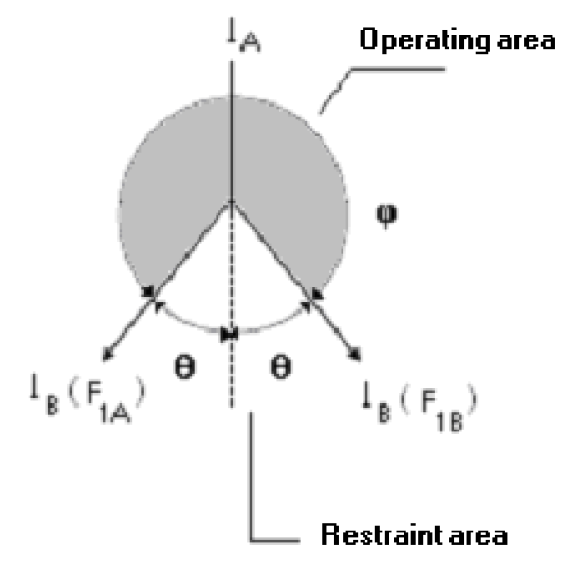

Figure(1b) : Principal of PC protection scheme

Figure (1) shows an example of fault detection by monitoring the difference between the phase-angles of the currents on both sides of the protected line as shown in (a) and the corresponding phasor diagram in (b). In figure $1 \mathrm{~b}$, the $\angle(\theta)$ is called the Stability Angle. In practice, a stability angle setting of $3 \mathrm{~ms}$ for a $60 \mathrm{~Hz}$ system allows for about 65 electrical degrees of blocking zone, provides sufficient security to prevent mal-tripping due to the reasons, indicated above and provides reliable tripping for all types of internal faults [9].

\section{Causes of CT Saturation Occurrence}

\subsection{High Fault Currents}

One of the reasons of saturation is increasing the fault current of the CT to values much higher than the saturation limit allowed for the CT. This current limit is determined by the accuracy limit factor (ALF) of the CT (According to IEC Classification of CT's Example: 5P20, 40VA

- $5 \mathrm{P}=$ class

- $20=\mathrm{ALF}$

At rated burden of 40VA, if current through CT is $20 \times$ nominal current, maximum measurement error will be $5 \%$ ).

\subsection{Offset in Primary Fault Current}

Another important cause of saturation is due to the direct current (DC) component of fault current. When a fault occurs the current is of the form:

$\mathrm{I}=\operatorname{Imax}[\sin (\omega \mathrm{t}+\psi-\theta)+\sin (\psi-\theta) \mathrm{e}(-\mathrm{rt} / \mathrm{L})]$

In which $\mathrm{r}$ and $\mathrm{L}$ are the resistance and the inductance of the primary circuit, $\theta$ is the 
phase-angle of the primary circuit, i.e. $\theta=\tan -1(\mathrm{~L} \omega / \mathrm{r})$, and $\psi$ is the time in radians after voltage zero at which the fault occurs. In equation (1) the first term is the symmetrical alternating current (AC) component and the second term is the DC component which starts at a maximum and decays exponentially. This of course refers to the primary current and hence does not include any CT characteristics because they affect only the CT secondary current. The formula indicates that the DC offset lasts longer if $\mathrm{r} / \mathrm{L}$ is small, e.g. in a highly inductive circuit such as a power system above $100 \mathrm{Kv}$. The maximum offset occurs when $\psi=90^{\circ}-\theta$. i.e. if the fault is initiated at the moment when $\mathrm{V}$ is going through zero $\left(\mathrm{V}_{\text {zero }}\right)$. However, it is almost impossible for a fault to occur near $\mathrm{V}_{\text {zero }}$ because over $95 \%$ of faults occur within $40^{\circ}$ before voltage maximum [10].

\subsection{High Burden on The Secondary of The CT}

Another contributor to saturation is the total burden of the secondary circuit, including the CT winding resistance as well as the resistance of the leads. In some substations, these leads may be quite long, which may require the use of larger than normal wire sizes or the use of parallel secondary wiring..

\subsection{The Remanent Flux in The Core of The CT}

The CT core may saturate prematurely at currents well below the normal saturation level due to the existence of remanent flux. Unfortunately, cold rolled silicon steel, which is favored nowadays because of its high saturating level, has high remanence so that the recent occurrence of a heavy fault may leave a remanent flux high enough to cause saturation when a second fault occurs [10]. Since circuit breakers tend to interrupt the current as it goes through zero, the amount of residual flux left in the CT core depends upon the phase-angle of its secondary burden. With a purely inductive burden the voltage will be at a maximum at the moment of current zero and the flux will be zero, hence there will be no remanent flux. With a purely resistive burden, the voltage will be zero at the moment of current zero and the flux will be at a maximum, hence there will be maximum remanent flux.

\section{Theoretical Basis of The Proposed Technique}

A concentrated analysis, for the saturated secondary current of the CT, have been done to find out the fundamental's magnitude and argument. Fourier series is being used for this task.

Assume the following waveform, which is shown in figure 2 as an example of a saturated signal to be analyzed using Fourier series, the following assumptions are considered:

1. AC saturation only is considered.

2. Remanance flux is neglected. 
3. A pure CT's resistive burden is connected

$$
f(x)=\left\{\begin{array}{cc}
\sin (x) & -\pi \leq x \leq \varphi-\pi \\
0 & \varphi-\pi<x<0 \\
\sin (x) & 0 \leq x \leq \varphi \\
0 & \varphi<x<\pi
\end{array}\right.
$$

Where $\varphi$ is the saturation angle, the angle at which the saturation occurs.

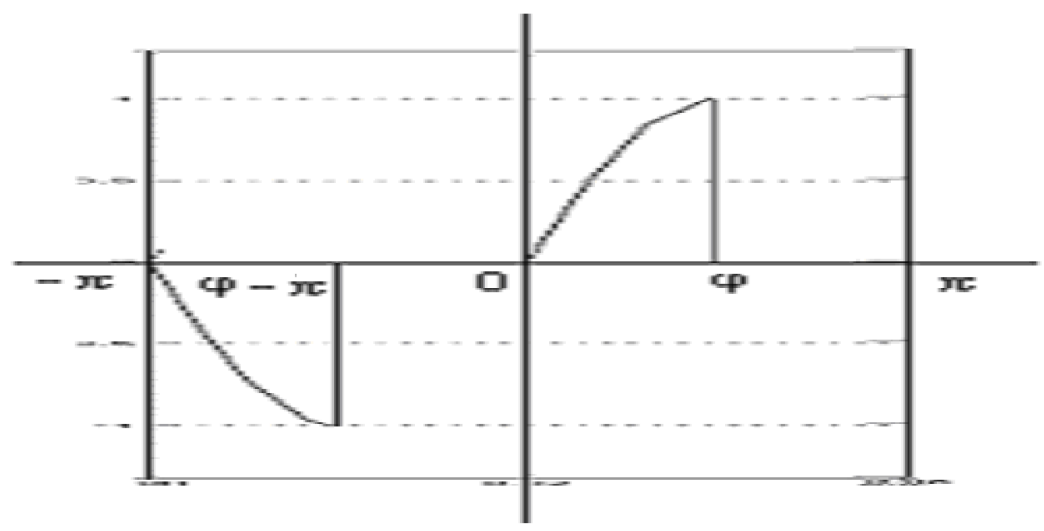

Figure (2) A saturated signal

Fourier coefficients:

$$
\begin{aligned}
& a_{o}=\frac{1}{2 \pi} \int_{-\pi}^{\pi} f(x) d x \\
& a_{n}=\frac{1}{\pi} \int_{-\pi}^{\pi} f(x) \cos (n x) d x \\
& b_{n}=\frac{1}{\pi} \int_{-\pi}^{\pi} f(x) \sin (n x) d x
\end{aligned}
$$


Fourier coefficients must be calculated to get the saturated signal in the form of

$$
f(x)=a_{o}+\left(a_{1} \cos (x)+b_{1} \sin (x)\right)+\left(a_{2} \cos (2 x)+b_{2} \sin (2 x)\right)+\left(a_{3} \cos (3 x)+b_{3} \sin (3 x)\right)+\ldots
$$

the integrations in (3), (4) and (5) must be calculate according to the function in (2) to find out a general formula to $a_{0}, a_{n}$ and $b_{n}$ respectively.

$$
\begin{aligned}
& a_{o}=\frac{1}{2 \pi} \int_{-\pi}^{\pi} f(x) d x \\
& a_{o}=\frac{1}{2 \pi}\left[\int_{-\pi}^{\varphi-\pi} \sin (x) d x+\int_{0}^{\varphi} \sin (x) d x\right] \\
& a_{o}=\frac{1}{2 \pi}\left[-\left.\cos (x)\right|_{-\pi} ^{\varphi-\pi}-\left.\cos (x)\right|_{0} ^{\varphi}\right] \\
& a_{o}=\frac{1}{2 \pi}[\cos (-\pi)-\cos (\varphi-\pi)+\cos (0)-\cos (\varphi)] \\
& a_{o}=\frac{1}{2 \pi}[-1+\cos (\varphi)+1-\cos (\varphi)]=0
\end{aligned}
$$

It is expected that $a_{o}=0$ because AC saturation is considered only.

$$
\begin{aligned}
& a_{n}=\frac{1}{\pi} \int_{-\pi}^{\pi} f(x) \cos (n x) d x \\
& a_{n}=\frac{1}{\pi}\left[\int_{-\pi}^{\varphi-\pi} \sin (x) \cos (n x) d x+\int_{0}^{\varphi} \sin (x) \cos (n x) d x\right] \\
& \left.a_{1}=\frac{1}{2 \pi}\left[\int_{-\pi}^{n}\langle\sin (n+1) x-\sin (n-1) x\rangle d x+\int \sin (n+1) x-\sin (n-1) x\right) d x\right]
\end{aligned}
$$




$$
\begin{aligned}
& a_{11}=\frac{1}{2 \pi}\left[\left.\frac{-\cos (n+1) x}{(n+1)}\right|_{-\pi} ^{2 \pi}+\left.\frac{\cos (n-1) x}{(n-1)}\right|_{-\pi} ^{-\pi}+\left.\frac{-\cos (n+1) x}{(n+1)}\right|^{n}+\left.\frac{\cos (n-1) x}{(n-1)}\right|_{n} ^{2}\right] ; n+1 \\
& \text { at } \quad n=1 \\
& a_{1}=\frac{1}{\pi}\left[\int_{-\pi}^{\varphi-\pi} \sin (x) \cos (x) d x+\int_{0}^{\varphi} \sin (x) \cos (x) d x\right] \\
& a_{1}=\frac{1}{2 \pi}\left[\left.\sin ^{2}(x)\right|_{-\pi} ^{\varphi-\pi}+\left.\sin ^{2}(x)\right|_{0} ^{\varphi}\right] \\
& a_{1}=\frac{1}{2 \pi}\left[\sin ^{2}(\varphi-\pi)-\sin ^{2}(-\pi)+\sin ^{2}(\varphi)-\sin ^{2}(0)\right] \\
& a_{1}=\frac{1}{2 \pi}\left[\sin ^{2}(\varphi)-0+\sin ^{2}(\varphi)-0\right] \\
& a_{1}=\frac{1}{\pi}\left[\sin ^{2}(\varphi)\right] \\
& b_{n}=\frac{1}{\pi} \int_{-\pi}^{\pi} f(x) \sin (n x) d x \\
& b_{n}=\frac{1}{\pi}\left[\int_{-\pi}^{\varphi-\pi} \sin (x) \sin (n x) d x+\int_{0}^{\varphi} \sin (x) \sin (n x) d x\right] \\
& b_{\mathrm{u}}=\frac{1}{2 \pi}\left[\int_{-\pi}^{\pi}(\cos (n-1) x-\cos (n+1) x) d x+\int_{0}^{n}(\cos (n-1) x-\cos (n+1) x) d x\right] \\
& b_{11}=\frac{1}{2 \pi}\left[\left.\frac{\sin (n-1) x}{(n-1)}\right|_{-\pi} ^{p-\pi}-\left.\frac{\sin (n+1) x}{(n+1)}\right|_{-\pi} ^{-\pi}+\left.\frac{\sin (n-1) x}{(n-1)}\right|_{0} ^{\infty}-\left.\frac{\sin (n+1) x}{(n+1)}\right|_{0} ^{1}\right] ; n+1 \\
& \text { at } n=1 \\
& b_{1}=\frac{1}{\pi}\left[\int_{-\pi}^{\varphi-\pi} \sin ^{2}(x) d x+\int_{0}^{\varphi} \sin ^{2}(x) d x\right] \\
& b_{1}=\frac{1}{2 \pi}\left[\int_{-\pi}^{\varphi-\pi}(1-\cos (2 x)) d x+\int_{0}^{\varphi}(1-\cos (2 x)) d x\right] \\
& b_{1}=\frac{1}{2 \pi}\left[\left.x\right|_{-\pi} ^{\varphi-\pi}-\left.\frac{\sin (2 x)}{2}\right|_{-\pi} ^{\varphi-\pi}+\left.x\right|_{0} ^{\varphi}-\left.\frac{\sin (2 x)}{2}\right|_{0} ^{\varphi}\right] \\
& b_{1}=\frac{1}{2 \pi}\left[\varphi-\pi+\pi-\left.\sin (x) \cos (x)\right|_{-\pi} ^{\varphi-\pi}+\varphi-0-\left.\sin (x) \cos (x)\right|_{0} ^{\varphi}\right]
\end{aligned}
$$




$$
\begin{aligned}
& b_{1}=\frac{1}{2 \pi}[p-\sin (\varphi-\pi) \cos (\varphi-\pi)+0+p-0-\sin (\varphi) \cos (\varphi)-0] \\
& b_{1}=\frac{1}{\pi}[\varphi-\sin (\varphi) \cos (\varphi)]
\end{aligned}
$$

Coefficients of the higher harmonics are not considered because the fundamental's argument is the most important factor in this paper. The fundamental of the saturated signal will be in the form:

$f_{1}(x)=\sqrt{a_{1}{ }^{2}+b_{1}{ }^{2}} \sin \left(x+\tan ^{-1}\left(\frac{a_{1}}{b_{1}}\right)\right)$

Fourier coefficients are calculated at different saturation angles and then substituted in equation (10) to find out the fundamental's phase angle. As shown in the following table:

Table 1. The fundamentals' equations at different values of $\varphi$.

\begin{tabular}{|c|c|}
\hline $\boldsymbol{\varphi}$ & Fundamentals' equations \\
\hline $180^{\circ}$ & $f_{1}(x)=\sin (x)$ \\
\hline $162^{\circ}$ & $f_{1}(x)=0.99 \sin (x+1.73)$ \\
\hline $144^{\circ}$ & $f_{1}(x)=0.96 \sin (x+6.6)$ \\
\hline $126^{\circ}$ & $f_{1}(x)=0.88 \sin (x+13.7)$ \\
\hline $108^{\circ}$ & $f_{1}(x)=0.74 \sin (x+22.54)$ \\
\hline $90^{\circ}$ & $f_{1}(x)=0.59 \sin (x+32.46)$ \\
\hline $72^{\circ}$ & $f_{1}(x)=0.42 \sin (x+43.26)$ \\
\hline $54^{\circ}$ & $f_{1}(x)=0.25 \sin (x+54.38)$ \\
\hline
\end{tabular}

The CT saturation of a pure resistive burden is considered the worst case of saturation, because it reduces the width of the secondary current waveform. This reduction in the waveform badly affects the phase and amplitude comparators. While, the CT saturation of a pure indutive burden reduces the amplitude of the secondary current waveform thus, its erroneous effect is limited to the amplitude comparator only. In Table 1 it is clearly noticed that there is a phase shift of the fundamental's phase-angle with respect to a pure sine wave, therefore a practical safety margin angle $(\varphi)$ should be taken into consideration to push the PC technique away from any risk of false decision. 


\section{Computer Numerical Simulation}

\subsection{The Simulation}

MATLAB-SIMULINK13 is chosen to build a model for a CT. The resulting saturated secondary current is analyzied using the following SIMULINK block:

Fourier analyzer:

The Fourier block performs a Fourier analysis of the input signal over a running window of one cycle of the fundamental frequency. First and second outputs return respectively the magnitude and phase-angle of the harmonic component specified. This block is used to calculate the fundamental component (magnitude and phase-angle) of the saturated current.

\subsection{Initiation of Saturation in Computer Simulation}

The CT is forced to go into saturation by two different methods:

1. Increasing the burden on its secondary.

2. Increasing its secondary current. (by increasing the primary fault current or decreasing the $\mathrm{CT}$ ratio).

The CT may get saturated due to one (or more) of the four mentioned reasons. However, the study concentrates only on two causes of saturation (increasing burden and secondary current), and it does not include DC saturation. Also, the residual flux is assumed to be zero. Therefore, the following two assumptions are considered:

1. AC saturation only is considered.

Remanence flux is neglected.

\subsection{Parameters Affecting Saturation}

Simulation is established to represent the CT getting saturated under different conditions to study the effect of the parameters affecting saturation. These parameters are:

1-Burden type.

2-Burden value.

3-CT magnetizing characteristics.

4-The ratio of the fault current to the CT rated current (I/In).

\section{4 Grouping The Simulation Cases}

To study the effect of each parameter of the above mentioned four parameters, many different cases are simulated (up to 116 cases). At each case, one parameter only is changed while the rest of parameters are kept constant. By this way, the influence of each parameter is clearly noted. So, the simulation cases are arranged into four groups, in each group, the effect of one parameter of the four above mentioned parameters is being studied. 
Those groups are:

1. Group (A): The effect of burden type is studied.

2. Group (B): The effect of the burden value is studied.

3. Group (C): The effect of the CT magnetizing characteristics is studied.

4. Group (D): The effect of I/In is studied.

\section{The Proposed Approach}

The proposed approach is based on the analysis of the results given in the simulation. The following is expected:

"As the CT's saturation increases, the magnitude of the secondary current decreases which leads to false operation of the differential relay. However, the PC could be used to assure the security of the operation based on fundamentals' phase-angles measurements of CT's secondary on both sides of the protected object". During external faults, the difference in phase-angle between currents' phase-angles on both sides of the protected object is equal to 180 . But, its value is zero during internal faults. If ideal CT's are considered. In practice, ideal CT's are not available therefore, error in current's phase-angle should be expected. Based on PC, it is possible to distinguish between internal and external faults when the current's phase-angle error is in acceptable margin during CT's saturation.

\section{Applying The Proposed Method on Busbar Protection}

A model for a busbar, as a part of a power system as shown in figure 3, is simulated by MATLAB-SIMULINK 13 to study the behavior of the busbar protection algorithm. The power system is exposed to different types of faults. To achieve the aim of this simulation, some CT's connected to the busbar under study, are saturated. The behavior of the developed differential protection relay is recorded. Figure 4 shows the waveform of a saturated CT.

Case Studies "External Faults With Saturation"

The following faults are simulated

Three phase to ground fault ( $3 \mathrm{ph}-\mathrm{G})$.

Double phase to ground fault $(2 \mathrm{ph}-\mathrm{G})$.

Single phase to ground fault $(1 \mathrm{ph}-\mathrm{G})$. 


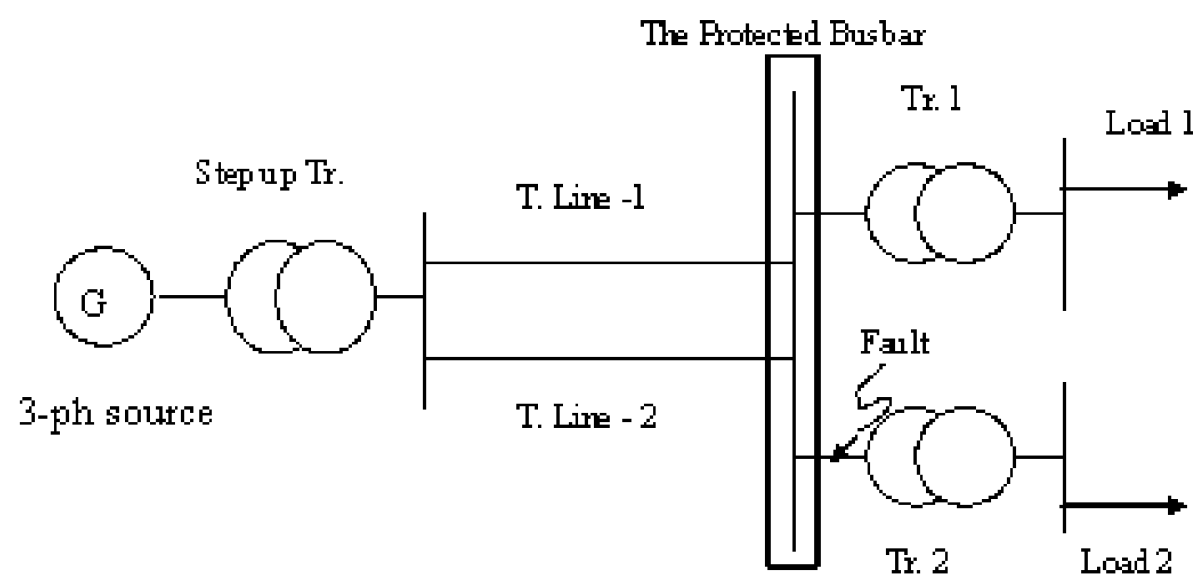

Figure 3: Single line diagram for the power system under study.

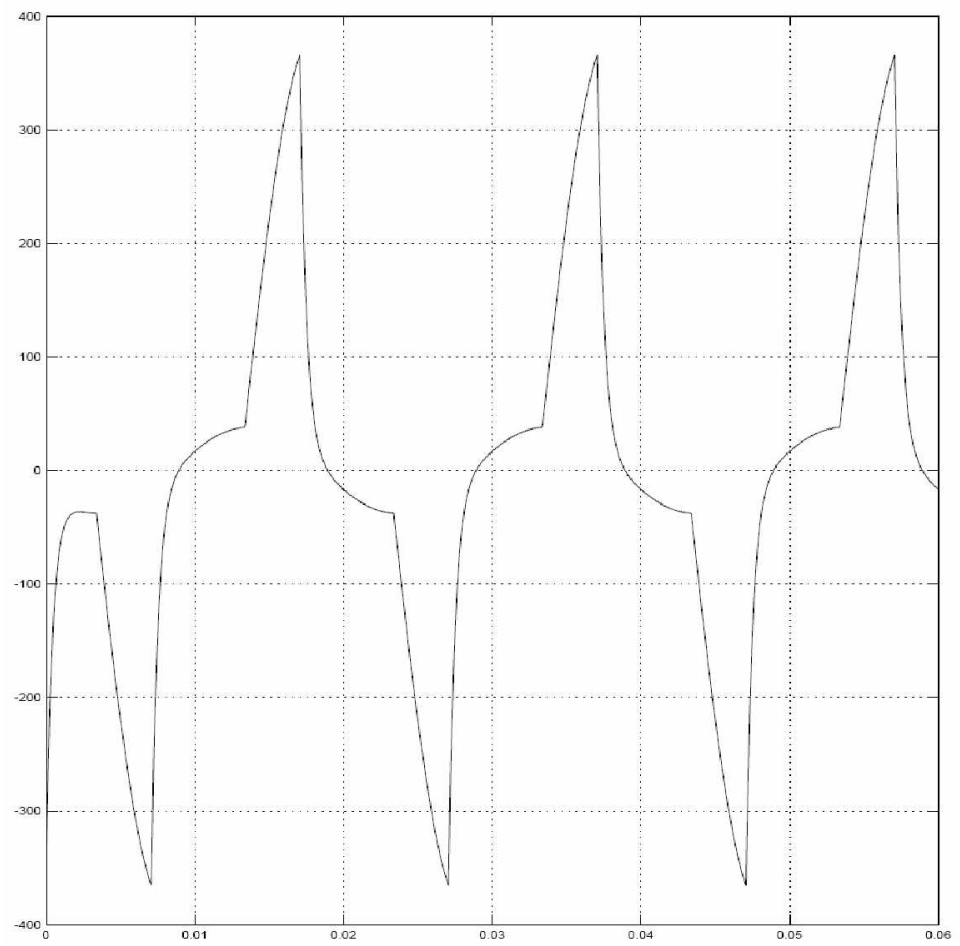

Figure4: The wave form of the secondary current of the saturated CT during 3-ph - to $G$ external fault. 
The proposed algorithm performance can be sumarized as follows:

1. Check if $I_{\text {Diff }}>k^{*} I_{\text {Rest }}$ or not "where $k$ is the stabilizing factor, $I_{\text {Diff }}$ is the differential current and $\mathrm{I}_{\text {Rest }}$ is the restraining current".

2. If $\mathrm{I}_{\text {Diff }}>\mathrm{k} \mathrm{I}_{\text {Rest }}$, then the proposed technique measures the fundamentals' phase-angles of the input and the output currents.

3. If the fundamentals' phase-angles difference between the input and the output currents is greater than $\beta$, so the saturation is detected and the relay is blocked.

4. Else, the difference will be less than $\beta$ then an internal fault is asserted and the trip signal will be issued.

Table (2) shows the results of applying the above steps as $\mathrm{k}=0.3$.

Table 2. The output results from the proposed algorithm during external faults with saturation

\begin{tabular}{|l|c|c|c|}
\hline \multicolumn{1}{|c|}{ Fault type } & $3 \mathrm{ph}-\mathrm{G}$ & $2 \mathrm{ph}-\mathrm{G}$ & $1 \mathrm{ph}-\mathrm{G}$ \\
\hline Differential current $\left(\mathrm{I}_{\text {Diff }}\right)$ & $214 \mathrm{~A}$ & $193.1 \mathrm{~A}$ & $105 \mathrm{~A}$ \\
\hline Restraining current $\left(\mathrm{I}_{\text {Rest }}\right)$ & $433.5 \mathrm{~A}$ & $414.9 \mathrm{~A}$ & $317.1 \mathrm{~A}$ \\
\hline Measured Stabilizing factor $\left(\mathrm{I}_{\text {Diff }} / \mathrm{I}_{\mathrm{Rest}}\right)$ & 0.49 & 0.478 & 0.33 \\
\hline Fault Current Value & $271.9 \mathrm{~A}$ & $252.5 \mathrm{~A}$ & $174.5 \mathrm{~A}$ \\
\hline Phase Shift Bet. Input \& Output currents & $136.3^{\circ}$ & $138.46^{\circ}$ & $151.03^{\circ}$ \\
\hline $\begin{array}{l}\text { Tripping Signal of Conventional Differential } \\
\text { Protection }\end{array}$ & 1 & 1 & 1 \\
\hline $\begin{array}{l}\text { Tripping Signal of the Developed Differential } \\
\text { Protection }\end{array}$ & 0 & 0 & 0 \\
\hline
\end{tabular}

It can be seen from the results of table (2) that the correct decision of the developed differential protection and the mal-operation of convential differential protection under CT saturation. 


\section{$\underline{\text { 8. Conclusion }}$}

This paper presents the use of the PC technique in overcoming the CT saturation problem. It can be extracted easily from the case studies that applying the proposed technique solves the problem of the mal-operation of the conventional percentage differential algorithm during external faults with saturation.

\section{References}

[1] MBCZ 10 Low Impedance Biased Differential Busbar Protection (Catalog), 1998.

[2] G. Hosemann VDE, H.M .Steigerweld VDE, "Modal Saturation detector for digital differential protection," IEEE Transactions on Power Delivery, vol. 8, no. 3, July 1993.

[3] Cesereo Fernendez, "An impedance-based CT saturation detection algorithm for busbar differential protection," IEEE Transactions on Power Delivery, vol. 16, no. 4, October 2001.

[4] Mohindar S. Sachdev, Tarlochan S. Sidhuand Harjeet Singh Gill, "A Busbar Protection Technique and Its Performance During CT Saturation and CT RatioMismatch," IEEE Transactions on Power Delivery, vol. 15, no. 3, July 2000.

[5] David C. Yu, James C. Cummins, Zhuding Wang, Hong-Jun Yoon, and Ljubomir A. Kojovic, "Correction of Current Transformer Distorted Secondary Currents Due to Saturation Using Artificial Neural Networks," IEEE Transactions on Power Delivery, vol. 16, no. 2, April 2001.

[6] Jiuping Pan, Khoi $\mathrm{Vu}$, and Yi Hu, "An Efficient Compensation Algorithm for Current Transformer Saturation Effects," IEEE Transactions on Power Delivery, vol. 19, no. 4, October 2004.

[7] Li Gtricun Liu Wanshun Jia Qingquan Teng Lin Cao Fengmei Li Ying, "A New Method of Detecting Current Transducer Saturation Based on Wavelet Transform," IEEE 2001.

[8] A.S.Mohammed, "Intelligent Protection System For Busbars", thesis, 2005.

[9] L60 Line Phase Comparison Relay (Catalog), 1998.

[10] A.R.Van C.Warrington, "Protective Relays, Their Theory \& Practice", Volume2, John Willey \& Sons, Inc., New York, 1969,p. 154, 178-179. 